\title{
O-GIcNAc-modified SNAP29 inhibits autophagy-mediated degradation via the disturbed SNAP29-STX17-VAMP8 complex and exacerbates myocardial injury in type I diabetic rats
}

\author{
LIN HUANG $^{1 *}$, PING YUAN $^{1 *}$, PENG YU $^{1}$, QILING KONG ${ }^{1}$, ZIXUAN XU $^{1}$, XIA YAN $^{2}$, \\ YANG SHEN $^{2,3}$, JUESHENG YANG ${ }^{4}$, RONG WAN ${ }^{2}, \mathrm{KUI} \mathrm{HONG}^{1-3}$, YANHUA TANG $^{4}$ and JINZHU HU ${ }^{1}$ \\ ${ }^{1}$ Department of Cardiovascular Medicine, ${ }^{2}$ The Jiangxi Key Laboratory of Molecular Medicine, \\ Departments of ${ }^{3}$ Genetic Medicine and ${ }^{4}$ Cardiovascular Surgery, The Second Affiliated Hospital of \\ Nanchang University, Nanchang, Jiangxi 330006, P.R. China
}

Received March 14, 2018; Accepted August 29, 2018

DOI: $10.3892 / \mathrm{ijmm} .2018 .3866$

\begin{abstract}
The O-linked $\beta$-N-acetylglucosamine (O-GlcNAc) modification and autophagy are associated with diabetic myocardial injury, however, the molecular mechanisms between the two processes remain to be fully elucidated. The purpose of the present study was to elucidate the molecular regulation of autophagy by O-GlcNAc-modified synaptosomal-associated protein 29 (SNAP29) in diabetic myocardial injury. A rat model of type I diabetes was established via intraperitoneal injection of streptozotocin (STZ; $55 \mathrm{mg} / \mathrm{kg}$ ). Significant increases in the O-GlcNAc modification and accumulation of the autophagy markers microtubule-associated protein 1 light chain $3 \alpha \mathrm{II} / \mathrm{I}$ and P62, which suggest that
\end{abstract}

Correspondence to: Dr Jinzhu Hu, Department of Cardiovascular Medicine, The Second Affiliated Hospital of Nanchang University, 1st Minde Road, Nanchang, Jiangxi 330006, P.R. China

E-mail: hujinzhu1983@sina.com

Dr Yanhua Tang, Department of Cardiovascular Surgery, The Second Affiliated Hospital of Nanchang University, 1st Minde Road, Nanchang, Jiangxi 330006, P.R. China

E-mail: tyh6565@163.com

*Contributed equally

Abbreviations: O-GlcNAc, O-linked $\beta$-N-acetylglucosamine; SNAP29, synaptosomal-associated protein 29; STZ, streptozotocin; TG, thiamet G; Don, 6-diazo-5-oxo-L-norleucine; NRCMs, neonatal rat cardiomyocytes; DM, diabetes mellitus; OGT, O-GlcNAc transferase; OGA, O-GlcNAcase; TEM, transmission electron microscopy; co-IP, coimmunoprecipitation; H\&E, hematoxylin and eosin; 3-MA, 3-methyladenine; LAMP2, lysosome-associated membrane protein 2; LC3, microtubule-associated protein 1 light chain $3 \alpha$; VAMP8, vesicle-associated membrane protein 8 ; STX17, syntaxin-17

Key words: O-linked $\beta$-N-acetylglucosamine, autophagy, diabetes mellitus, myocardial injury, SNAP29 autophagic flux is inhibited, were observed in rats 8 weeks following STZ induction. Subsequently, the selective O-GlcNAcase inhibitor, thiamet $\mathrm{G}$, increased the level of O-GlcNAc modification, which further disrupted autophagic flux; deteriorated cardiac diastolic function, as indicated by an increased left ventricular filling peak velocity/atrial contraction flow peak velocity ratio shown by echocardiography; and exacerbated myocardial abnormalities, as characterized by cardiomyocyte disorganization and fat and interstitial fibrosis accumulation. By contrast, 6-diazo-5-oxo-L-norleucine, an inhibitor of glucosamine fructose-6-phosphate aminotransferase isomerizing 1, acted as an O-GlcNAc antagonist and reduced the level of O-GlcNAc modification, which maintained autophagic flux and improved cardiac diastolic function. In vitro, high glucose $(25 \mathrm{mM})$ was used to stimulate primary neonatal rat cardiomyocytes (NRCMs). Consistent with the myocardium of diabetic rats, it was also shown in the NRCMs that O-GlcNAc modification of SNAP29 negatively regulated autophagic flux. The application of the short hairpin RNA interference lysosome-associated membrane protein (LAMP2) and the autophagy inhibitor 3-methyladenine demonstrated that high glucose inhibited autophagy-mediated degradation rather than affected the initial stage of autophagy. Finally, co-immunoprecipitation was used to determine the role of the O-GlcNAc-modified substrate protein SNAP29, which acted as an SNAP29-syntaxin-17 (STX17)-vesicle-associated membrane protein 8 (VAMP8) complex during disease progression. The present study is the first, to the best of our knowledge, to demonstrate that SNAP29 is an O-GlcNAc substrate and that an increase in O-GlcNAc-modified SNAP29 inhibits SNAP29-STX17-VAMP8 complex formation, thereby inhibiting the degradation of autophagy and exacerbating myocardial injury in type I diabetic rats.

\section{Introduction}

The cardiovascular complications of diabetes mellitus (DM), including heart failure and arrhythmia, lead to high rates of disability and mortality (1-3). The molecular mechanism of myocardial injury in DM is complicated, and further 
investigation of this pathogenic mechanism is beneficial to further improving the prognosis of DM.

The posttranslational modification of O-linked $\beta-\mathrm{N}$-acetylglucosamine (O-GlcNAc) regulates proliferation, differentiation, apoptosis, autophagy and other physiological and pathological processes in a variety of cells, including cardiomyocytes (4-9). Two genes encode the enzymes for O-GlcNAcylation; O-GlcNAc transferase (OGT) catalyzes the addition of UDP-GlcNAc to target proteins, whereas O-GlcNAcase (OGA) removes the GlcNAc moiety from proteins (10). Accordingly, the selective O-GlcNAcase inhibitor, thiamet $\mathrm{G}$ (TG), effectively increases the level of O-GlcNAc modification. The concerted actions of the two enzymes allow the O-GlcNAc modification to be a dynamic post-translational modification; 6-diazo-5-oxo-L-norleucine (Don) is an inhibitor of glucosamine fructose-6-phosphate aminotransferase isomerizing 1 , which is an enzyme that controls the flux of glucose into the hexosamine pathway and catalyzes the formation of glucosamine 6-phosphate. In 2013, a study published in the Journal Nature (11) first applied Don as a specific O-GlcNAc antagonist, which showed that $5 \mu \mathrm{M}$ of Don effectively inhibited the level of O-GlcNAc in vitro. Several studies have subsequently used Don as an inhibitor of O-GlcNAc. Based on these previous publications $(12,13)$, Don was selected as a selective O-GlcNAc inhibitor in the present study to clarify the role of O-GlcNAc modified SNAP29 in cardiomyopathy in type I diabetic rats. Previous studies $(11,14)$ have found that the acute inhibition of O-GlcNAc inhibits premature ventricular complexes activated by diabetic hyperglycemia through O-GlcNAc- and $\mathrm{Ca}^{2+} /$ calmodulin-dependent protein kinase II-dependent pathways. Additionally, the dysregulation of O-GlcNAcylation is likely to be important in the rat cardiac mitochondrial dysfunction associated with diabetes, which was reported by Banerjee et al (15). Reportedly (16), an excessive level of O-GlcNAc modification in a DM animal model led to the worsening of cardiac function, whereas a reduction in O-GlcNAc levels improved cardiac function. However, the mechanism between the O-GlcNAc modification and the deterioration of cardiac function remains to be fully elucidated.

Autophagy is a dynamic process, which is regulated by multiple genes and molecular signals. The process of autophagosome formation, transport of the autophagic substrate to lysosomes, and autophagosomal degradation in lysosomes is termed autophagic flux. Autophagy is an important metabolic pathway for maintaining eukaryotic cell homeostasis in response to starvation, inflammation, and hypoxia/reoxygenation injury. Autolysosomes mainly eliminate aging organelles and misfolded proteins to provide energy for cells. In the normal myocardium, autophagy is maintained at a low level (17). However, energy metabolism of the myocardium is disturbed in DM, and autophagy can be overactivated or inhibited (18), although how autophagic flux causes myocardial injury in DM remains to be elucidated. Specifically, no reports have determined whether myocardial injury is influenced by interactions between the O-GlcNAc modification and autophagic flux in type I DM.

Synaptosomal-associated protein 29 (SNAP29) is mainly involved in the localization and fusion of organelle membranes in the cytoplasm (19). Several studies (20-22) have reported that SNAP29 is involved in mediating membrane fusion between autophagosomes and lysosomes, and forms a complex with syntaxin-17 (STX17) and vesicle-associated membrane protein 8 (VAMP8) to participate in the autophagy process. A previous study (23) showed that SNAP29 is modified as a protein substrate by $\mathrm{O}-\mathrm{GlcNAc}$ in Nematodes. However, whether the O-GlcNAc modification of SNAP29 regulates autophagic flux in a mammalian disease model remains to be elucidated.

\section{Materials and methods}

Reagents. The primary antibody mouse anti-O-GlcNAc (RL2; cat.no. RB229146; 1:1,000) was purchased from Life Company (New York, USA). The anti-OGT (cat. no. ab177941; 1:1,000), anti-LAMP2 (cat. no. ab125068 1:1,000), anti-SNAP29 (cat. no. ab181151; 1:1,000), anti-VAMP8 (cat. no. ab76021; 1:1,000), anti-STX17 (cat. no. ab116113; 1:1,000) and anti-OGA (cat. no. ab105217; 1:1,000) antibodies were obtained from Abcam (Cambridge, USA). The anti-Beclin1 (cat. no. D40C5; 1:1,000) antibody was obtained from Cell Signaling Technology, Inc. (Danvers, MA, USA). The anti-P62 (cat. no. 18420-1-AP; 1:1,000) and anti-Tubulin (cat. no. 66240-1-Ig; 1:1,000) antibodies were obtained from ProteinTech Group, Inc. (Chicago, IL, USA). Protein A/G beads were obtained from Abmart, Inc. (Shanghai, China). Anti-LC3B antibody (cat. no. L7543; 1:1,000), STZ (cat. no. SD130), TG (cat. no. SML0244), and Don (cat. no. D2141) were purchased from Merck KGaA (Darmstadt, Germany). The RNAi-LAMP2 sequences were purchased from Santa Cruz Biotechnology, Inc. (Dallas, TX, USA). The three sequence pairs of RNAi-LAMP2 were as follows: Sequence 1 forward, 5'-GAAGUUCUUAUAUGU GCAATT-3' and reverse, 5'-UUGCACAUAUAAGAACUU CTT-3'; sequence 2 forward, 5'-GGCAGGAGUACUUAUUCU ATT-3' and reverse, 5'-UAGAAUAAGUACUCCUGCCTT-3'; sequence 3 forward, 5'-CUGCAAUCUGAUUGAUUAUU-3' and reverse, 5'-TAAACACTGCTTGACCACC-3'. The most effective sequence was selected according to the interference efficiency detected using western blot analysis.

Animal experiments. All animal experiments were approved by the Animal Ethics and Experimentation Committee of Nanchang University (Nanchang, China) and were performed in accordance with the 'Guide for the Care and Use of Laboratory Animals' (revised 1996). The experimental protocol was approved by the Second Affiliated Hospital of Nanchang University. All experimental animals were purchased from Hunan Slack Jingda Experimental Animal Co., Ltd. (Hunan, China). A total of 50 adult male Sprague Dawley (SD) rats were purchased at 8-weeks old (weight range, 180-200 g), and kept in a specific pathogen-free conditions at room temperature $20-24^{\circ} \mathrm{C}$ and humidity $50-60 \%$. The experiment began after 1 week of acclimation. Rats were provided with adequate food and drinking water and maintained on a 12/12-h light-dark cycle to mimic the normal biorhythm of the rats.

Animal model establishment and treatment. All rats were intraperitoneally (i.p) injected with streptozotocin (STZ) at a dose of $55 \mathrm{mg} / \mathrm{kg}$ (24). Glucose concentrations were monitored 
using a commercial blood glucose-monitoring kit (Accusoft; Roche Diagnostics, Laval, QC, Canada) using blood samples obtained from the tail vein of nonfasting animals prior to and following the STZ injection, and immediately prior to the start of the experiment. Animals with tail vein blood glucose levels $>16.7 \mathrm{mmol} / \mathrm{l}$ were considered to have DM $(11,25)$. Control animals (vehicle) received the same volume of sodium citrate (pH 4.4, i.p.).

The rats were randomly divided into five groups $(n \geq 6$ in each group). The Vehicle group comprised the solvent control group injected with sodium citrate (pH 4.4; i.p). The STZ group, as the diabetic group, comprised rats injected with STZ i.p. at a dose of $55 \mathrm{mg} / \mathrm{kg}$. At 4 weeks post-STZ injection, rats were randomly divided into three groups: The STZ+Don group comprised STZ rats injected with the O-glycosylation inhibitor, Don (Sigma-Aldrich; Merck KGaA) at a dose of $5 \mathrm{mg} / \mathrm{kg}$, i.p. The STZ+TG group comprised STZ rats injected with O-glycosylation agonist TG (Sigma-Aldrich; Merck KGaA) at a dose of $25 \mathrm{mg} / \mathrm{kg}$, i.p. The $\mathrm{STZ}+\mathrm{NaCl}$ group comprised STZ rats injected with $0.9 \% \mathrm{NaCl}$, i.p. Don, $\mathrm{TG}$, and $\mathrm{NaCl}$ injected on a 2-day interval. All the rats were sacrificed as outlined in the experimental protocols.

Doppler echocardiography. Transthoracic echocardiography was performed prior to sacrifice at the indicated time points, as previously described $(26,27)$. Briefly, the rats were anesthetized with $2.0 \%$ isoflurane via inhalation. The chest was shaved, and the rats were placed in a supine position. Echocardiographic images were obtained by placing the transducer against the chest. M-mode echocardiograms of the left ventricle (LV) were recorded at the level of the papillary muscle using a commercially available echocardiographic system, Vevo2100, equipped with a $17-\mathrm{MHz}$ transducer (Visualsonics, Toronto, ON, Canada). The passive LV filling peak velocity $(\mathrm{E}, \mathrm{mm} / \mathrm{sec})$ and atrial contraction flow peak velocity $(\mathrm{A}, \mathrm{mm} / \mathrm{sec})$ were acquired from the mitral valve Doppler flow images in the apical four-chamber view.

Transmission electron microscopy (TEM), hematoxylin and eosin $(H \& E)$ staining and Masson staining. All the cardiac tissues were fixed with $10 \%$ buffered formalin, and a region of the ventricle was fixed with $2.5 \%$ glutaraldehyde for analysis by TEM. The TEM, H\&E staining and Masson staining were performed by Google Biological Technology (Wuhan, China). The quantitative results were analyzed with Image Pro Plus 6.0 software (Media Cybernetics, Inc., Rockville, MD, USA).

\section{Cell culture and reagents}

Isolation and treatment of neonatal rat cardiac myocytes $(N R C M s)$. The NRCMs were derived from newborn rats within 3 days according to a conventional protocol described previously $(28,29)$. Newborn SD rats were purchased from Hunan Slack Jingda Experimental Animal Co., Ltd. The mice were kept with their mothers for 3 days after birth, and after 1 day of stabilization in specific pathogen-free conditions at room temperature $20-24^{\circ} \mathrm{C}$ and humidity $50-60 \%$, they were subjected to cardiomyocyte isolation. The cells were cultured in DMEM supplemented with $15 \%$ fetal bovine serum (FBS) (both from Gibco; Thermo Fisher Scientific, Inc., Waltham, MA, USA) and $1 \%$ penicillin-streptomycin at $37^{\circ} \mathrm{C}$ with $5 \%$ carbon dioxide $\left(\mathrm{CO}_{2}\right)$. The NRCMs were exposed to DMEM containing $25 \mathrm{mM}$ glucose to mimic hyperglycemia injury, and the normal-glucose group was exposed to DMEM containing $5 \mathrm{mM}$ glucose.

The NRCMs were treated with drugs and randomly divided into eight groups: Normal glucose (Vehicle group), normal glucose+Don (Don group), normal glucose+TG (Sigma-Aldrich; Merck KGaA), normal glucose+3-methyladenine (3-MA group), high glucose (Glu group), high glucose+Don (Don+Glu group), high glucose+TG (TG+Glu group) and high glucose+3-MA (3-MA+Glu). In the present study, the dose and time schedule of drug application were as follows: In vitro, NRCMs were treated with DMEM containing $25 \mathrm{mM}$ glucose for $24 \mathrm{~h}$ to mimic hyperglycemic injury, Don $(40 \mu \mathrm{M})$ for $24 \mathrm{~h}$, TG $(5 \mu \mathrm{M})$ for $24 \mathrm{~h}$, or 3 -MA $(5 \mathrm{mM})$ for $24 \mathrm{~h}$.

Plasmid and adenovirus transfection. All cells were seeded in 6-well plates 1 day prior to transfection at a density of $1 \times 10^{5}$ cells/well. The cells were infected with either the OGT knockdown adenovirus (sh-OGT), OGT-overexpression adenovirus (ad-OGT) or OGA-overexpression adenovirus (ad-OGA) at an MOI of 50, and re-incubated with DMEM supplemented with $10 \%$ FBS $6 \mathrm{~h}$ following transfection. After $48 \mathrm{~h}$, the transfection efficiency was detected via fluorescence microscopy and western blot analysis.

Small interfering (si)RNA oligonucleotides specific for LAMP2A were synthesized by Santa Cruz Biotechnology, Inc. All-star negative siRNA was used as a negative control. The siRNA sequences targeting the indicated proteins were as follows: si-LAMP2, forward 5'-CUGCAAUCUGAUUGAUUA UU-3' and reverse 5'-TAAACACTGCTTGACCACC-3'. The NRCMs were seeded in 6 -well plates $\left(1 \times 10^{5}\right.$ cells/well). After $24 \mathrm{~h}$, the cells were transfected with $50 \mathrm{pmol}$ of siRNA/well using Lipofectamine 3,000 (cat. no. 1781682, Invitrogen; Thermo Fisher Scientific, Inc.) according to the manufacturer's protocol.

Western blot analysis and coimmunoprecipitation (co-IP). Proteins were extracted with a mixed lysis buffer containing a proteasome inhibitor (cat. no. RL2274422, Thermo Fisher Scientific, Inc.) and phenylmethanesulfonyl fluoride (cat. no. ST506; Beyotime Institute of Biotechnology, Shanghai, China). Western blot analysis was performed as previously described (29). Briefly, following protein extraction, the protein concentration was detected using a bicinchoninic acid assay. A total of $100 \mu \mathrm{g}$ protein/lane lysates were separated by $5-12 \%$ SDS-PAGE and subsequently transferred onto polyvinylidene difluoride membranes. Following blocking with Blocking reagent (cat.no. P0023B; Beyotime Institute of Biotechnology) at room temperature for $1 \mathrm{~h}$, the membranes were incubated overnight at $4{ }^{\circ} \mathrm{C}$ with the aforementioned primary antibodies and the corresponding goat anti-mouse (1:5,000; cat. no. SA00001-1; ProteinTech Group, Inc.) or goat anti-rabbit (1:5,000; cat. no. SA00001-15; ProteinTech Group, Inc.) horseradish peroxidase-conjugated $\operatorname{IgG}(\mathrm{H}+\mathrm{L})$ secondary antibodies at room temperature for $1 \mathrm{~h}$. The protein bands were visualized using ECL western blotting reagent (cat. no. 32209; Thermo Fisher Scientific, Inc.). The band intensities were quantified using Image Lab 4.0.1 software (Bio-Rad Laboratories, Inc., Hercules, CA, USA). The NRCMs, treated 
as mentioned above, were harvested, and co-IP was performed as previously described (29). Briefly, $400 \mu \mathrm{g}$ of total cell lysate was incubated with $4 \mu \mathrm{g}$ of primary antibody for the co-IP. The remaining cell lysates were incubated with rabbit IgG at $4^{\circ} \mathrm{C}$ overnight as a negative control. Following incubation of the cells with the primary antibodies, the lysates were mixed with protein A/G beads overnight. The co-IP samples were separated by SDS-PAGE and analyzed using western blot analysis as aforementioned.

Statistical analysis. The results are expressed as the mean \pm standard error of the mean of at least three independent experiments, and the differences between two groups were analyzed using Student's t-test. When more than two groups were compared, one-way analysis of variance followed by Tukey's post hoc test was used. Statistical analyses were performed using Graph Pad Prism 5 (GraphPad Software, Inc., La Jolla, CA, USA). $\mathrm{P}<0.05$ was considered to indicate a statistically significant difference.

\section{Results}

Myocardial injury is accompanied by an increase in O-GlcNAc modification and inhibited autophagic flux in rats at 8 weeks following STZ induction. Compared with the vehicle group, the STZ-induced rats manifested as hyperglycemic (30.49 \pm 0.6211 , vs. $5.881 \pm 0.2036 \mathrm{mmol} / 1, \mathrm{P}<0.01)$, a loss of body weight, and an increased heart/body weight ratio beginning at 4 weeks post-STZ administration (data not shown). Diabetic myocardial injury is mainly manifested as early diastolic dysfunction characterized by an abnormal E/A ratio. The echocardiographic results showed that the E/A ratio decreased significantly in rats at 8 weeks post-STZ induction compared with the vehicle rats $(1.30 \pm 0.04$, vs. $1.68 \pm 0.05$, $\mathrm{P}<0.01$; Fig. 1A); no significant difference in cardiac systolic function was observed, however, the cardiac structure was expanded (LVEF\%: $81.47 \pm 2.92$, vs. $72.92 \pm 4.45, \mathrm{n} \geq 6, \mathrm{P}>0.05$ ). Compared with the vehicle group, the STZ group had higher levels of cardiomyocyte disorganization and fat accumulation as detected by $H \& E$ staining. Additionally, the diabetic rats showed increased myocardial fibrosis on Masson staining (Fig. 1B and C).

Additionally, the results of the western blot analysis showed a significant increase in the O-GlcNAc modification and the expression of OGT, and a reduction in the expression of OGA in the myocardium of rats at 8 weeks post-STZ induction compared with the vehicle rats (Fig. 1D; $\mathrm{P}<0.05$ ). The expression levels of the autophagy markers Beclin1 (30) and LC3II/I were increased significantly in the STZ group. The expression levels of SQSTM1/P62 and lysosome-associated membrane protein LAMP2 (31) were also significantly increased. These results indicated that the autophagy-mediated degradation process and autophagic flux were inhibited in rats at 8 weeks post-STZ induction (Fig. $1 \mathrm{E} ; \mathrm{P}<0.05$ ). As the gold standard for the identification of autophagy, TEM showed a marked increase in autophagosomes with a double-membrane structure in the STZ group (Fig. 1F). These results suggested that the significant increase in myocardial O-GlcNAc modification was simultaneously accompanied by autophagic flux inhibition in the DM rat hearts.
Increased O-GlcNAc modification in vivo aggravates myocardial injury and inhibits autophagic flux in type I DM rats. To further clarify the effect of O-GlcNAc modification on myocardial injury in type I DM rats, the selective O-GlcNAcase inhibitor TG and the glutamine antagonist Don were used to investigate the effects of O-GlcNAc modification on cardiac function and myocardial structure in type I DM rats. The preliminary results (data not shown) demonstrated that, at 4 weeks post-STZ induction, no significant difference was observed in the level of O-GlcNAc modification, myocardial structure or the levels of autophagy, compared with those in the vehicle rats, however, the E/A ratio was marginally decreased $(\mathrm{P}<0.05)$. Therefore, drug intervention in the STZ-induced rats was started at 4 weeks. The results showed that TG significantly enhanced the heart/body weight ratio of the rats at 8 weeks post-STZ induction. By contrast, Don significantly mitigated the above phenotype in type I DM rats. The E/A ratio in the STZ+TG group was significantly higher than that in the Vehicle group $(2.28 \pm 0.14$, vs. $1.67 \pm 0.067, \mathrm{P}<0.05)$ and the $\mathrm{STZ}+\mathrm{NaCl}$ group $(2.28 \pm 0.14$, vs. $1.29 \pm 0.081, \mathrm{P}<0.01)$, which suggested that the $\mathrm{LV}$ pressure was increased and the diastolic function was deteriorated further. In addition, the E/A ratio was increased in the STZ+Don group compared to that in the $\mathrm{STZ}+\mathrm{NaCl}$ group (1.62 \pm 0.13 , vs. $1.29 \pm 0.081, \mathrm{P}<0.05)$, which suggested that the cardiac diastolic function was improved in the DM rats following treatment with the O-GlcNAc inhibitor (Fig. 2A). No significant difference in cardiac systolic function was observed among the STZ-induced groups. Additionally, the agonist TG aggravated myocardial disorganization and fat deposition, as detected by H\&E staining, and intercellular fibrosis, as assessed by Masson staining, in the heart tissues of STZ rats. Furthermore, Don significantly rescued the abnormal myocardial structure in type I DM rats (Fig. 2B and C). The above results suggested that the increased level of O-GlcNAc modification aggravated myocardial injury in the type I DM rats.

To investigate the correlation between myocardial O-GlcNAc modification and autophagy in type I DM rats, Don and TG were administered in vivo. Western blot analysis was used to detect the levels of myocardial O-GlcNAc modification and autophagy in each group. Following drug administration, the level of O-GlcNAc modification and the expression of OGT were significantly increased in the STZ+TG group compared with those in the $\mathrm{STZ}+\mathrm{NaCl}$ group $(\mathrm{P}<0.05)$, whereas the expression of OGA was notably decreased $(\mathrm{P}<0.05)$. Compared with that in the $\mathrm{STZ}+\mathrm{NaCl}$ group, the expression of OGA was significantly increased in the STZ+Don group $(\mathrm{P}<0.05)$, and the level of O-GlcNAc modification and expression of OGT were significantly decreased (Fig. 2D, P<0.05). Compared with the STZ+NaCl group, the expression levels of LC3II/I, P62 and LAMP2 were markedly increased in the STZ+TG group, and the expression of Beclin1 was significantly decreased $(\mathrm{P}<0.05)$, which suggested that the O-GlcNAc modification level was increased and that autophagy-mediated degradation was inhibited. Following treatment with Don, the expression levels of LC3II/I and P62 were decreased, and the expression of LAMP2 was significantly increased (Fig. 2E, $\mathrm{P}<0.05)$. In addition, the TEM results showed that autophagosomes were increased in the $\mathrm{STZ}+\mathrm{TG}$ and $\mathrm{STZ}+\mathrm{NaCl}$ groups, compared with those in the vehicle and STZ+Don groups, respectively (Fig. 2F). The above results indicated that 
A

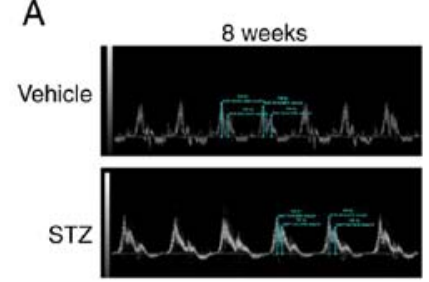

B

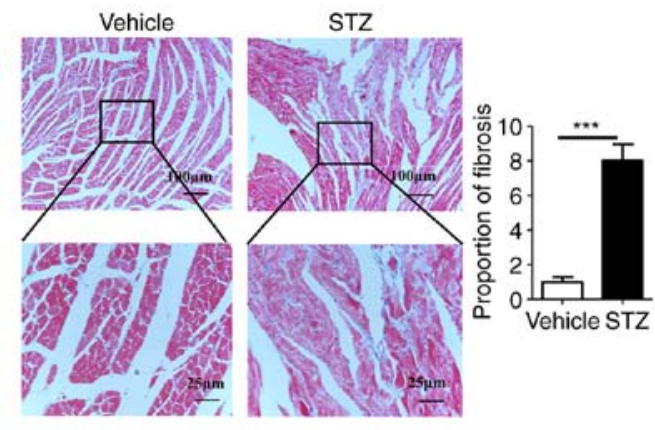

C

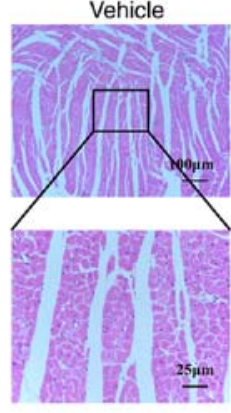

$\mathrm{STZ}$

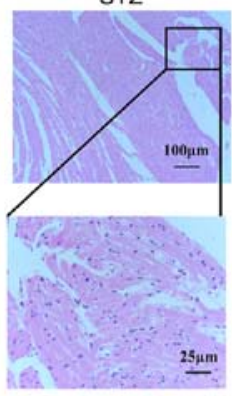

D

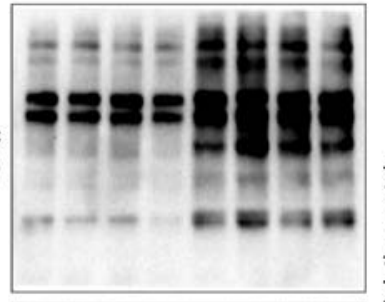

O-GICNAc

(RL2)

E

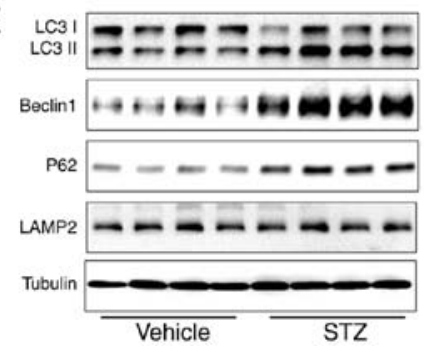

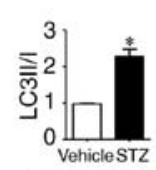

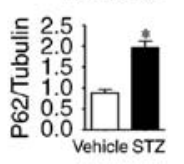

OGT

OGA

Tubulin
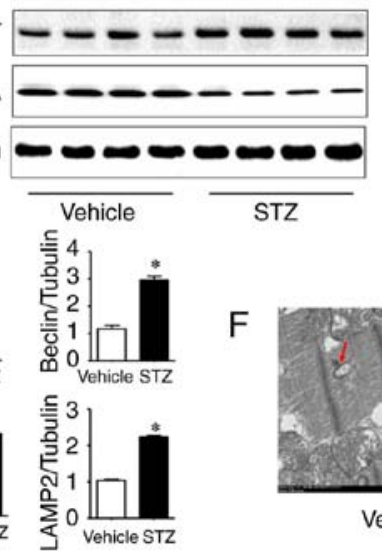

$\mathrm{F}$

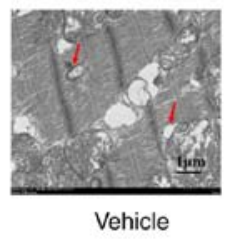

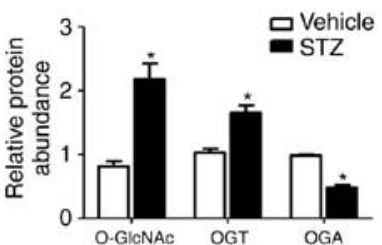

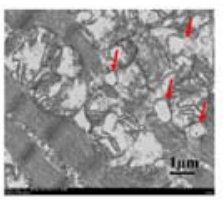

STZ

Figure 1. Myocardial injury is accompanied by an increase in O-GlcNAc modification and inhibited autophagic flux in rats at 8 weeks post-STZ induction. (A) M-mode echocardiography shows the left ventricular diastolic function as the ratio of E/A in rats at 8 weeks post-STZ induction. Morphological changes in the myocardium were assessed by (B) Masson staining and (C) hematoxylin and eosin staining (scale bar=100 and $25 \mu \mathrm{m}$ ). (D) Protein was extracted from heart tissue, and the expression levels of O-GlcNAc (RL2), OGT and OGA were detected by western blot analysis; (E) Expression levels of LC3II/I, LAMP2, Beclin-1 and P62 in each group were detected by western blot analysis in the vehicle group and the 8-week STZ-induced group. (F) Transmission electron microscopy shows autophagosomes, characterized by a double-layer membrane structure, as indicated with the red arrows (scale bar=1 $\mu \mathrm{m}$ ). Data are expressed as the mean \pm standard error of the mean $(\mathrm{n} \geq 6)$. ${ }^{*} \mathrm{P}<0.05$ and ${ }^{* *} \mathrm{P}<0.01$, vs. Vehicle group. Tubulin was the loading control. O-GlcNAc, $\mathrm{O}-$ linked $\beta$-N-acetylglucosamine; STZ, streptozotocin; OGT, O-GlcNAc transferase; OGA, O-GlcNAcase; LAMP2, lysosome-associated membrane protein 2; LC3, microtubule-associated protein 1 light chain $3 \alpha$; E/A, left ventricular filling peak velocity/atrial contraction flow peak velocity.

myocardial O-GlcNAc modification was increased in the heart of type I DM rats and that the degradation of autophagosomes was significantly increased when O-GlcNAc modification was inhibited, thereby ensuring the progression of autophagy.

Increased O-GlcNAc modification inhibits autophagic flux in NRCMs under high-glucose conditions. The NRCMs were subjected to high-glucose stimulation $(25 \mathrm{mM})$ to further verify the impact of the changes in the level of O-GlcNAc modification on autophagy. The administration of TG increased the level of O-GlcNAc modification in the normal- and high-glucose environments, whereas the inhibitor Don exerted a potent inhibitory effect on O-GlcNAc modification compared with that in the control group (Fig. 3A). Consistent with the results in the animal experiments, autophagic flux was significantly inhibited in the NRCMs under high-glucose conditions, as demonstrated by the increased expression levels of LC3II/I,
Beclin1, P62, and LAMP2. In addition, the O-GlcNAc modification agonist TG inhibited autophagic flux but increased the level of O-GlcNAc modification (Fig. 3B). By contrast, the O-GlcNAc modification inhibitor Don significantly reduced O-GlcNAc levels and promoted autophagic flux.

Aside from changing the level of O-GlcNAc modification by Don and TG administration, NRCMs were separately transfected with adenoviruses that either suppressed the expression of OGT (sh-OGT) or overexpressed OGA (ad-OGA) to reduce the level of O-GlcNAc modification. Western blot analysis was used to detect the levels of autophagy under normal- and high-glucose conditions. However, regardless of the glucose level, sh-OGT and ad-OGA significantly reduced the levels of O-GlcNAc modification (Fig. 3C, P<0.05). Measurement of autophagy-related markers showed that, under high-glucose stimulation, the expression levels of LC3II/I, Beclin1, $\mathrm{P} 62$, and LAMP 2 were significantly increased $(\mathrm{P}<0.001)$, 

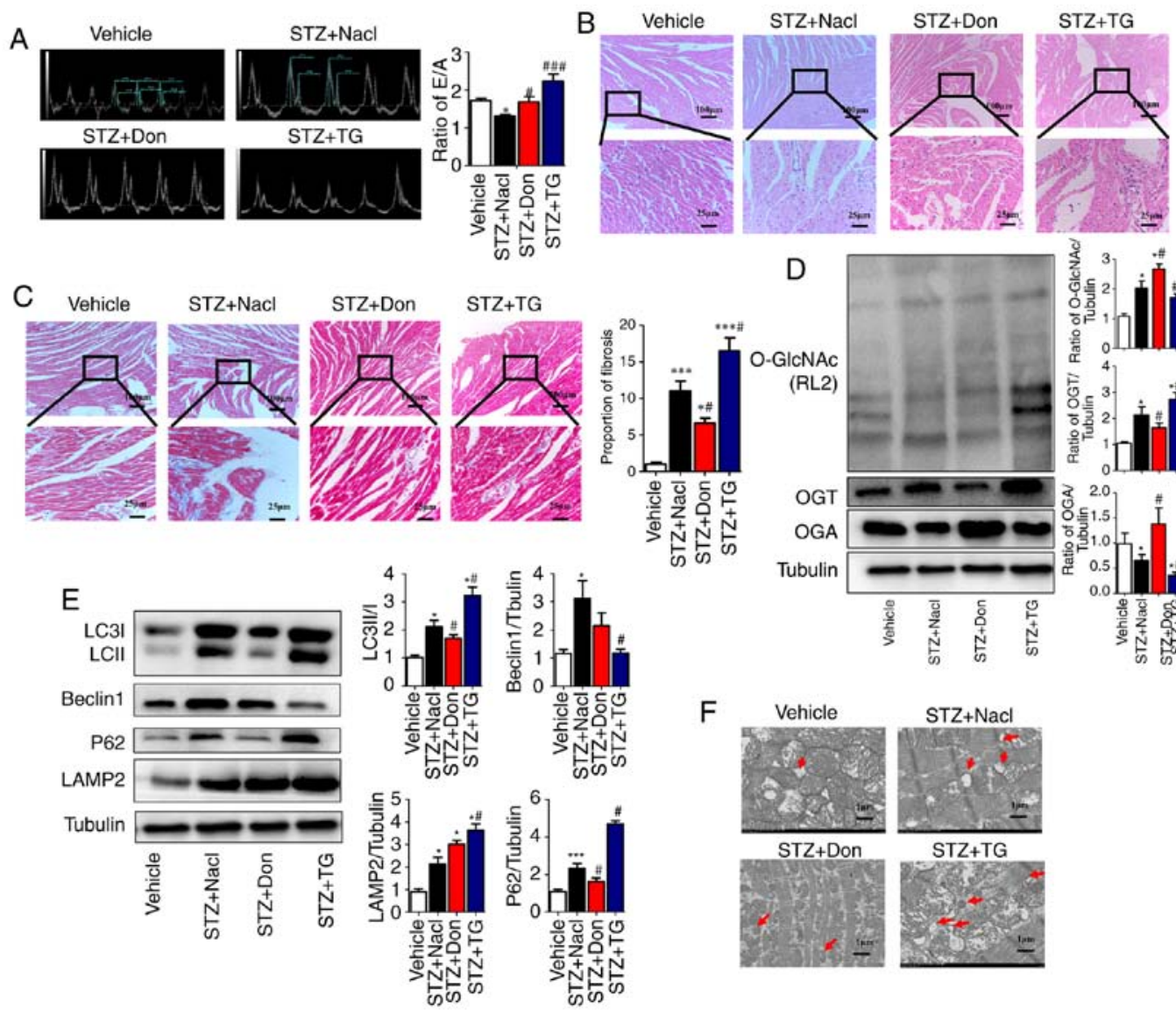

$\mathrm{D}$
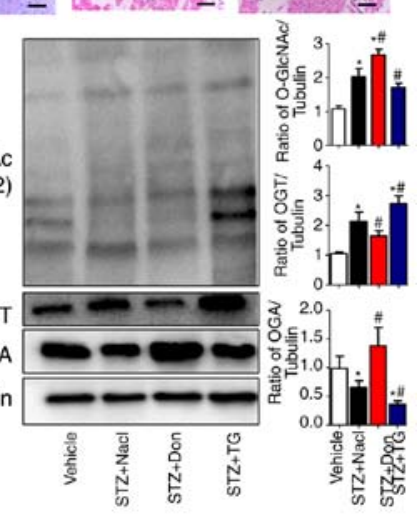

Figure 2. Increased O-GlcNAc modification in vivo inhibits autophagic flux and aggravates myocardial function in type I DM rats. Rats were randomly divided into five groups ( $\mathrm{n} \geq 6$ in each group) for treatment: Vehicle, STZ, STZ+NaCl, STZ+Don, and STZ+TG. (A) M-mode echocardiography showed the left ventricular diastolic function as the ratio of E/A. Morphological changes in the myocardium were assessed by (B) hematoxylin and eosin staining and (C) Masson staining (scale bar=100 and $25 \mu \mathrm{m}$ ); (D) Expression levels of O-GlcNAc (RL2), OGA and OGT in each group were detected by western blot analysis. (E) Western blot analysis was used to detect the expression of autophagy markers LC3II/I, Beclin1, P62, and LAMP2. Data are expressed as the mean \pm standard error of the mean $(\mathrm{n} \geq 6)$. ${ }^{*} \mathrm{P}<0.05$ and ${ }^{* * * *} \mathrm{P}<0.001$, vs. Vehicle group; ${ }^{\#} \mathrm{P}<0.05$ and ${ }^{\# \# \#} \mathrm{P}<0.001$, vs. STZ+NaCl group. Tubulin was the loading control. (F) Transmission electron microscopy showed the autophagosomes, which are characterized by a double-layer membrane structure, as indicated by red arrows (scale bar=1 $\mu \mathrm{m}$ ). O-GlcNAc, O-linked $\beta$-N-acetylglucosamine; STZ, streptozotocin; Don, 6-diazo-5-oxo-L-norleucine; TG, thiamet G; OGT, O-GlcNAc transferase; OGA, O-GlcNAcase; LAMP2, lysosome-associated membrane protein 2; LC3, microtubule-associated protein 1 light chain 3 $\alpha$; E/A, left ventricular filling peak velocity/atrial contraction flow peak velocity.

which indicated that autophagy was significantly increased (Fig. 3D). OGT interference or OGA overexpression resulted in a significant decrease in autophagy and autophagic flux degradation in NRCMs under normal- and high-glucose conditions. Therefore, consistent with the results of the aforementioned drug intervention, interference with the O-GlcNAc modification promoted autophagic flux in the NRCMs under normal- and high-glucose conditions.

High glucose levels inhibit the degradation stage of autophagy. To clarify the specific stage of autophagy that is affected by high glucose in NRCMs, the autophagy inhibitor 3-MA and LAMP2-interference sequences were used. The optimal interfering sequence of LAMP2 was selected according to the efficiency of transfection, which was verified by western blot analysis (data not shown). The results showed that, under the normal-glucose condition, disrupted expression of LAMP2 in NRCMs was associated with increased levels of Beclin1, LC3II/I, and P62 ( $\mathrm{P}<0.01)$. Under the high-glucose condition, LAMP2 interference did not increase the levels of Beclin1 or LC3II/I, which indicated that high glucose levels affected the degradation process of autophagic flux (Fig. 4A). To further validate this hypothesis, the autophagy inhibitor 3-MA was used to treat NRCMs at different glucose levels. In normal glucose levels, the expression levels of LC3II/I and P62 decreased following 3-MA treatment, which was in line with the function of 3-MA as an inhibitor of class III phosphoinositide 3-kinase in the initial stage of autophagy. In the high glucose-induced NRCMs, the expression levels of LC3II/I and P62 decreased with 3-MA treatment compared with those in NRCMs induced with high glucose alone, which indicated that high glucose levels did not affect the initial stage of autophagy (Fig. 4B). The above results showed that high glucose levels inhibited autophagy-mediated degradation rather than the initial stage of autophagy.

O-GlcNAc modification of SNAP29 inhibits SNAP29-STX17-VAMP8 complex formation and inhibits autophagy-mediated degradation. To reveal the specific role of SNAP29 in the regulation of autophagic flux, co-IP was used to detect O-GlcNAc-modified SNAP29 and observe differences in the formation of the SNAP29-STX17-VAMP8 

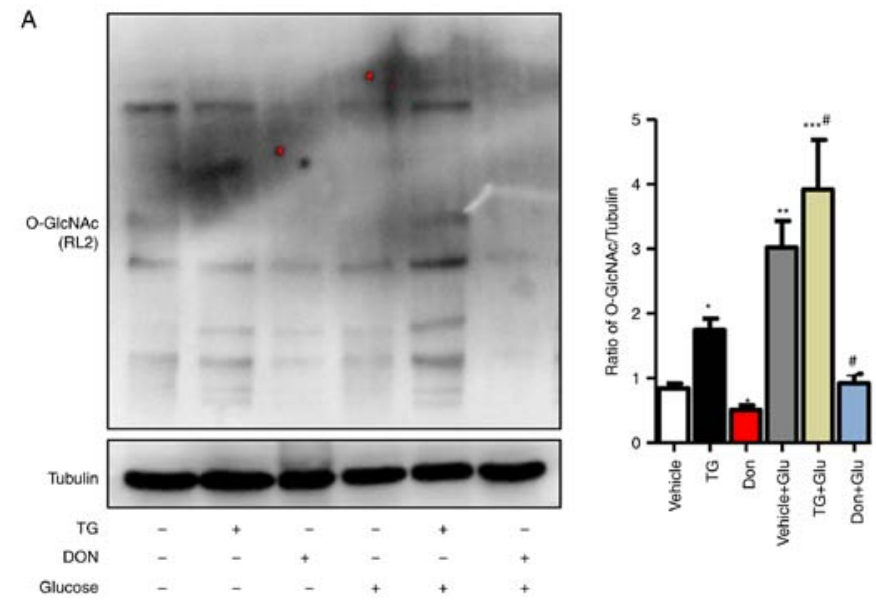

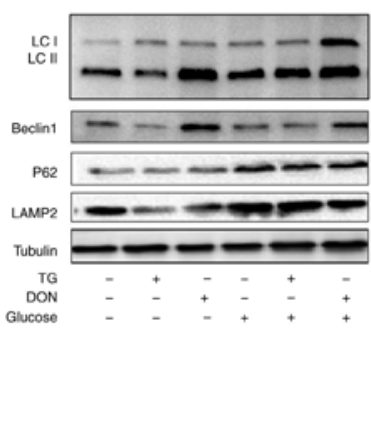

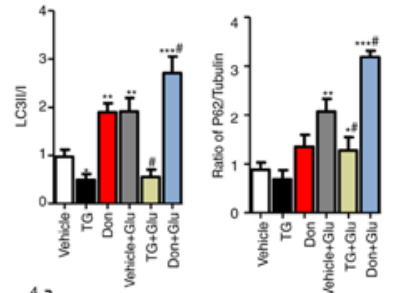

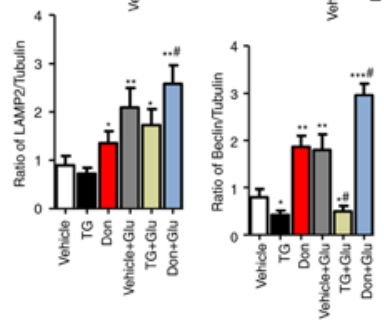

D
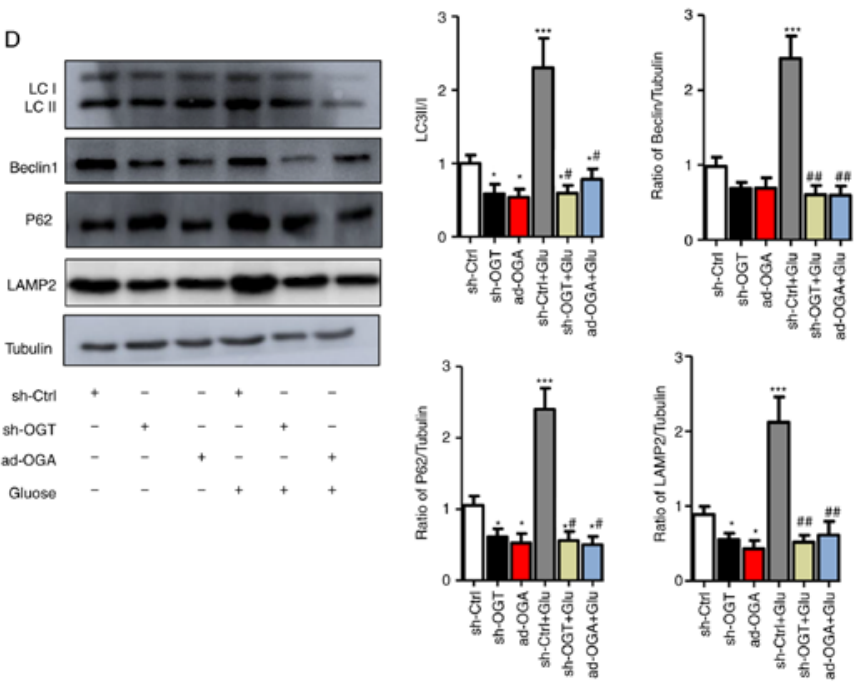

Figure 3. Increased O-GlcNAc modification inhibits autophagic flux in NRCMs under high-glucose conditions. NRCMs were exposed to high glucose (25 mM) and were treated with TG $(5 \mu \mathrm{M})$ or Don $(40 \mu \mathrm{M})$ for $24 \mathrm{~h}$. (A) Supernatants were extracted, and the expression of O-GlcNAc (RL2) was analyzed by western blot analysis. (B) Expression levels of LAMP2, Beclin-1, P62, and LC3II/I in each group were detected by western blot analysis. NRCMs were transfected with sh-OGT or ad-OGA for $48 \mathrm{~h}$ and were exposed to high glucose (25 mM, Glu group) for $24 \mathrm{~h}$. (C) Supernatants were extracted, and the expression levels of O-GlcNAc (RL2), OGT, and OGA were analyzed by western blot analysis. (D) Expression levels of LAMP2, Beclin-1, P62, and LC3II/I in each group were detected by western blot analysis. Data are expressed as the mean \pm standard error of the mean $(\mathrm{n} \geq 6)$. ${ }^{*} \mathrm{P}<0.05,{ }^{* * *} \mathrm{P}<0.01$ and ${ }^{* * * *} \mathrm{P}<0.001$, vs. Vehicle group; ${ }^{\#} \mathrm{P}<0.05$ and ${ }^{\# \#} \mathrm{P}<0.01$, vs. Glu group. Tubulin was the loading control. NRCMs, neonatal rat cardiomyocytes; O-GlcNAc, O-linked $\beta$-N-acetylglucosamine; STZ, streptozotocin; sh-OGT, OGT-knockdown adenovirus; ad-OGA, OGA-overexpression adenovirus; Ctrl, control; Glu, glucose; Don, 6-diazo-5-oxo-L-norleucine; TG, thiamet G; OGT, O-GlcNAc transferase; OGA, O-GlcNAcase; LAMP2, lysosome-associated membrane protein 2; LC3, microtubule-associated protein 1 light chain 3 $\alpha$.

complex. In the rat heart tissues, SNAP29, but not VAMP8 or STX17, was modified by O-GlcNAc (Fig. 5A). In addition, the level of O-GlcNAc modification in SNAP29 was markedly increased in the STZ group, whereas binding of the SNAP29-STX17-VAMP8 complex was decreased significantly (Fig. 5B-D). High-glucose stimulation did not alter the relevant expression levels of SNAP29, VAMP8 or STX17 in the NRCMs. These results suggested that only O-GlcNAc-modified SNAP29 was involved in the regulation of autophagy. Under continuous high-glucose stimulation in the presence of the agonist TG, O-GlcNAc modification of SNAP29 was significantly increased, and the interactions among SNAP29, STX17 and VAMP8 were significantly decreased in the NRCMs (Fig. 6A-C). Following incubation with the inhibitor Don, sh-OGT and ad-OGA, the level of O-GlcNAc-modified SNAP29 was decreased, but the forma- tion of the SNAP29-VAMP8-STX17 complex was increased (Fig. 6D-F). The above results suggested that O-GlcNAc modification of SNAP29 affected the degradation of autophagic flux via negative regulation of SANP29-VAMP8-STX17 complex formation.

\section{Discussion}

To the best of our knowledge, the present study is the first to examine the regulatory mechanism between O-GlcNAc-modified SNAP29 and autophagy in the progression of diabetic myocardial injury in an animal model. As shown in Fig. 7, under continuous high-glucose stimulation, the increased O-GlcNAc modification of SNAP29 effectively inhibited the formation of the SANP29-STX17-VAMP8 complex, which acts as the mediator of autophagosome and 
A

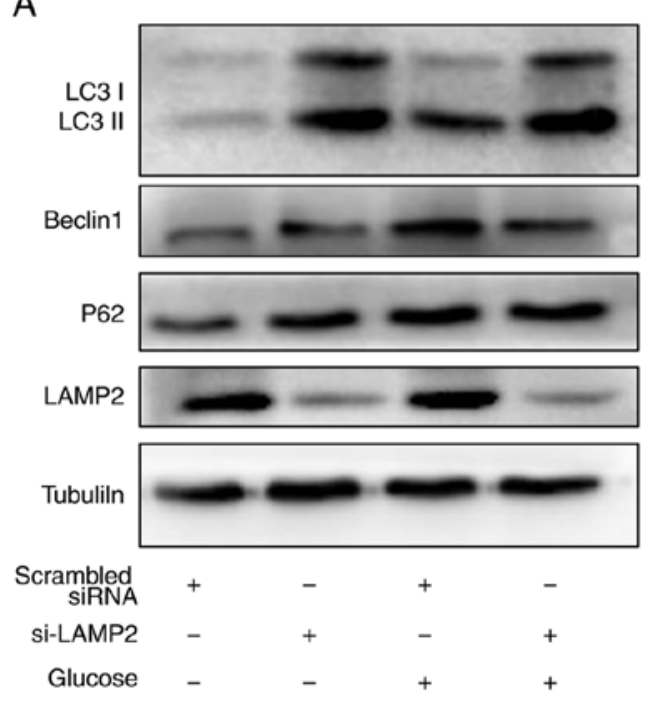

B

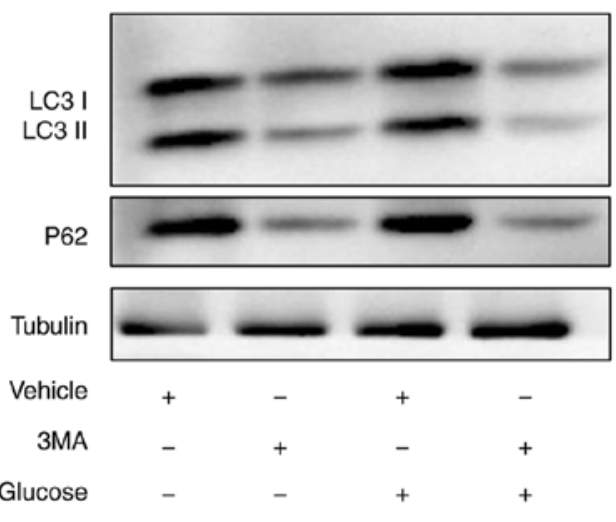

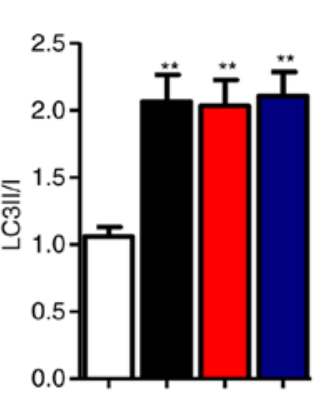
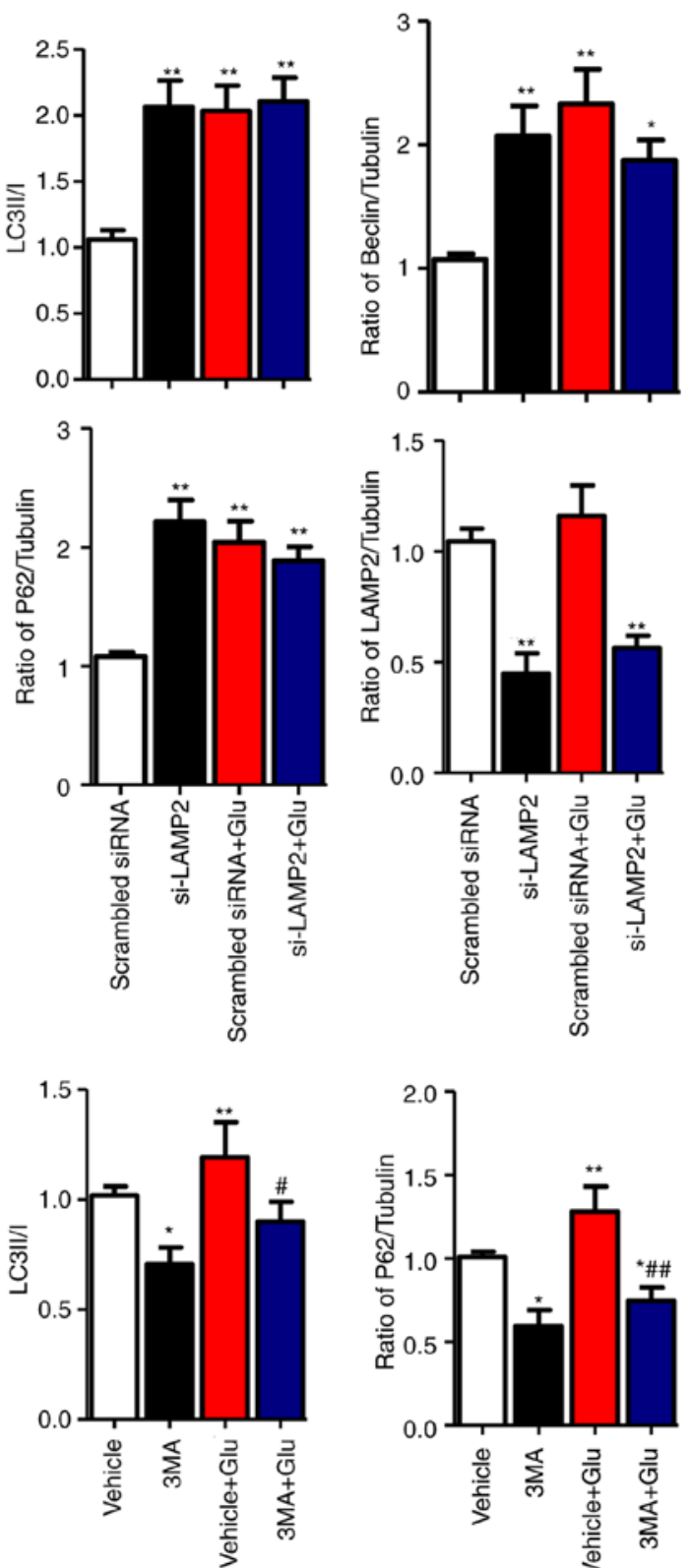

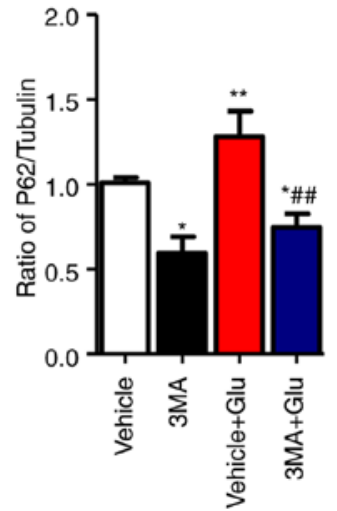

Figure 4. High glucose inhibits the degradation stage of autophagy. (A) NRCMs were transfected with LAMP2 siRNA for 48 h and were exposed to high glucose for another $24 \mathrm{~h}$. The expression of LAMP2, Beclin-1, P62, and LC3II/I in each group was detected by western blot analysis. (B) NRCMs were pretreated with 3-MA $(5 \mathrm{mM})$ for $24 \mathrm{~h}$ and with high glucose for another $24 \mathrm{~h}$; the expression levels of P62 and LC3II/I in each group were detected by western blot analysis. Data are expressed as the mean \pm standard error of the mean $(\mathrm{n} \geq 6)$. ${ }^{*} \mathrm{P}<0.05$ and ${ }^{* *} \mathrm{P}<0.01$, vs. Vehicle group; ${ }^{\#} \mathrm{P}<0.05$ and ${ }^{\# \#} \mathrm{P}<0.01$, vs. Glu group. Tubulin was the loading control. NRCMs, neonatal rat cardiomyocytes; O-GlcNAc, O-linked $\beta$-N-acetylglucosamine; Glu, glucose; siRNA, small interfering RNA; LAMP2, lysosome-associated membrane protein 2; LC3, microtubule-associated protein 1 light chain 3 $\alpha$; 3-MA, 3-methyladenine.

lysosome fusion and inhibits autophagy-mediated degradation, resulting in myocardial injury in the type I DM heart.

The O-GlcNAc modification is affected by multiple factors, including intracellular glucose and lipid metabolism. Disruption of O-GlcNAc modification exerts an important biological effect on diabetes, various types of cancer, neurodegeneration, and the cardiovascular system (32). Enhanced O-GlcNAc modification regulates a variety of molecular signaling pathways to severely impair conduction-contractile coupling, the uptake and release of $\mathrm{Ca}^{2+}$, and energy metabolism of the mitochondria in cardiomyocytes. Consistent with previous studies, the results of the present study demonstrated that O-GlcNAc modification was gradually enhanced over time in type I DM rat hearts.
The activation of O-GlcNAc modification aggravated the disordered cardiomyocyte arrangement, fat accumulation, and intercellular fibrosis, and the deterioration of cardiac diastolic function, whereas inhibition of the O-GlcNAc modification improved cardiac function and ameliorated the abnormalities in myocardial structure. No significant difference in cardiac systolic function (predominantly LVEF) was observed between the Vehicle and STZ groups at 8 weeks post-STZ injection. Due to the different strategies of STZ injection, there are differences between the results of the present study and those of other studies. In general, cardiac dysfunctions are observed in STZ-induced diabetic rats, including diastolic and systolic dysfunction, which are dependent on the dose of STZ 
A
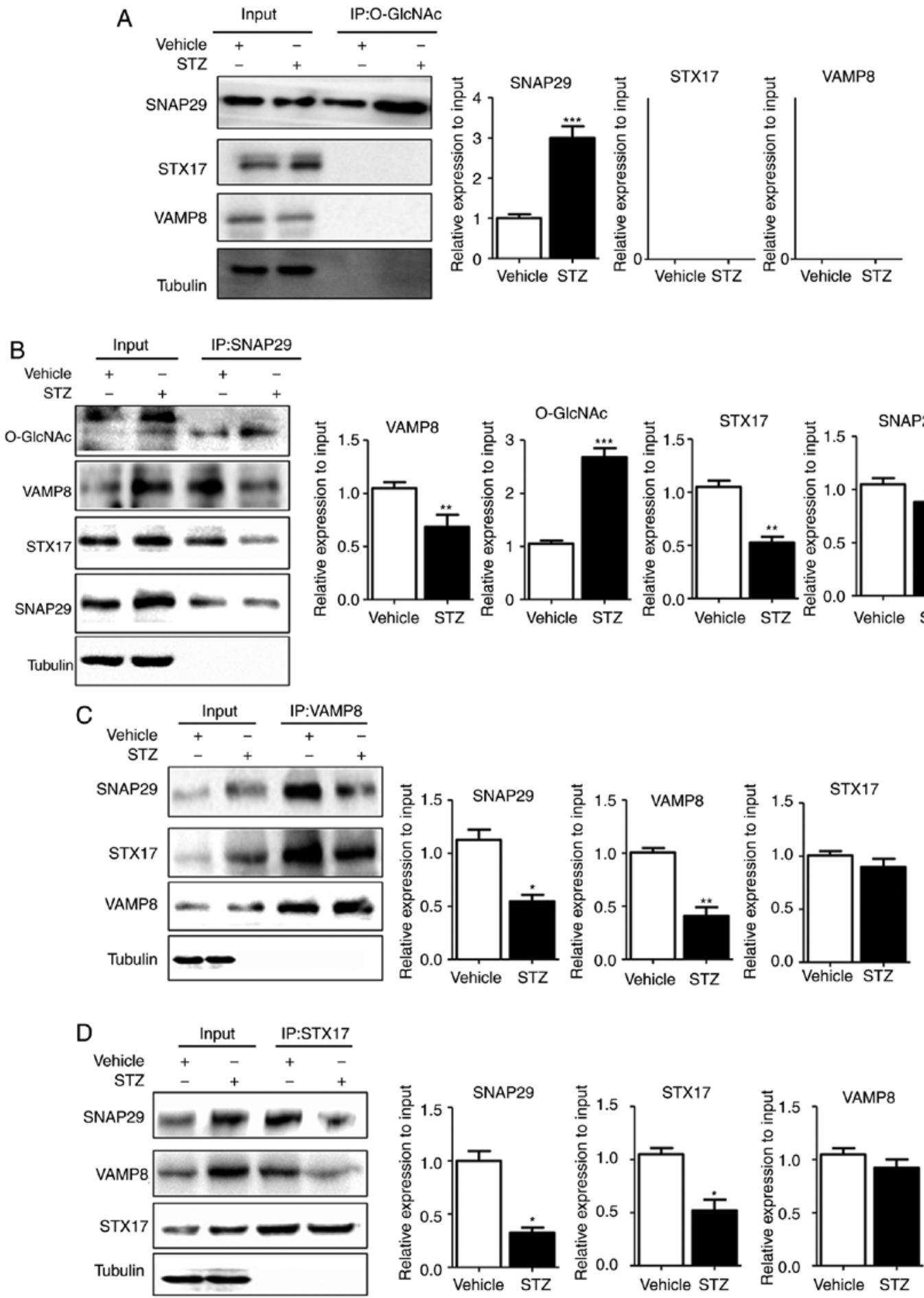

Figure 5. SNAP29 is modified by O-GlcNAc and form a complex with STX17 and VAMP8. In heart tissues from vehicle and streptozotocin-induced rats, the interaction between the O-GlcNAc modification and SNAP29-VAMP8-STX17 was evaluated by co-IP. (A) O-GlcNAc antibody was used as the known target to pull down the complex proteins SNAP29, VAMP8 and STX17, and Tubulin was used as the loading control. (B) Similarly, SNAP29 antibody was used as pull down the O-GlcNAc, VAMP8 and STX17, SNAP29 and Tubulin were used as the loading control; (C) VAMP8 antibody was applied to pull down the complex SNAP29 and STX17, VAMP8 and Tubulin were used as the loading control. (D) STX17 antibody was used to pull down the complex SANP29 and VAMP8, both STX17 and Tubulin acted as the loading control. $\left({ }^{*} \mathrm{P}<0.05,{ }^{, *} \mathrm{P}<0.01,{ }^{* * * *} \mathrm{P}<0.001\right.$ vs. vehicle group). O-GlcNAc, O-linked $\beta$-N-acetylglucosamine; SNAP29, synaptosomal-associated protein 29; vesicle-associated membrane protein 8; STX17, syntaxin-17; Ctrl, control; co-IP, coimmunoprecipitation.

administration and the duration of time following induction of STZ. Studies $(33,34)$ have reported that a high dose of STZ injection $(65 \mathrm{mg} / \mathrm{kg})$ resulted in a decreased systolic function 8 weeks post-STZ administration. Generally $(35,36)$, it was not until 11-12 weeks post-STZ injection that the cardiac systolic function was decreased.
Low-level autophagy is an important method by which cardiomyocyte homeostasis is maintained. The change in autophagy in the DM rats was mainly affected by abnormal energy metabolism caused by high glucose. The overactivation or inhibition of any step of the process of autophagy can cause myocardial injury. Under different conditions, diabetic 

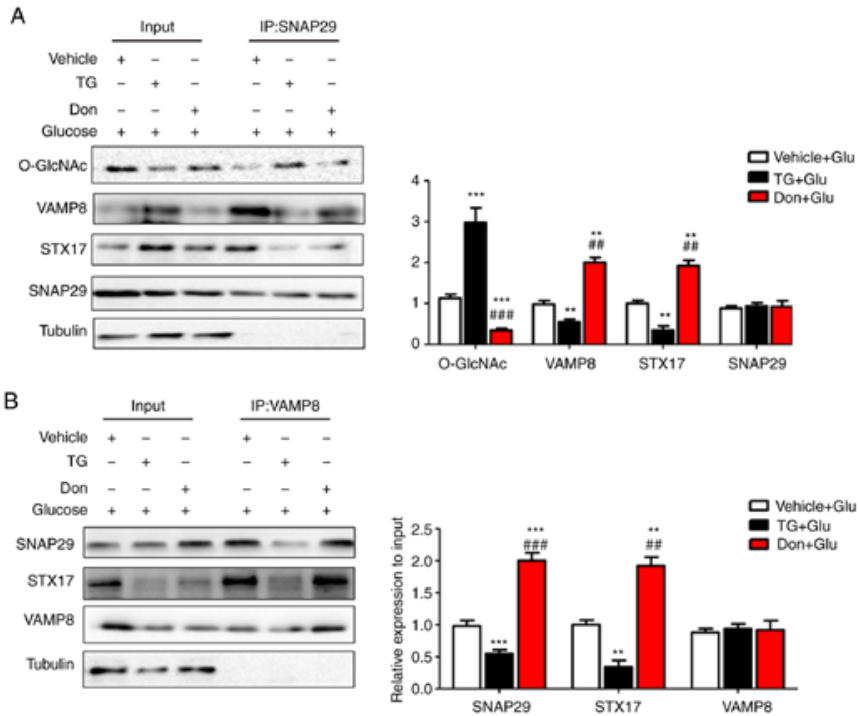

$\mathrm{E}$
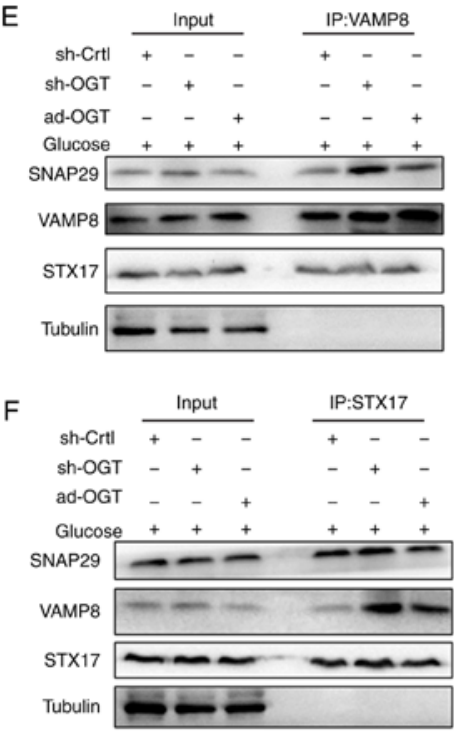
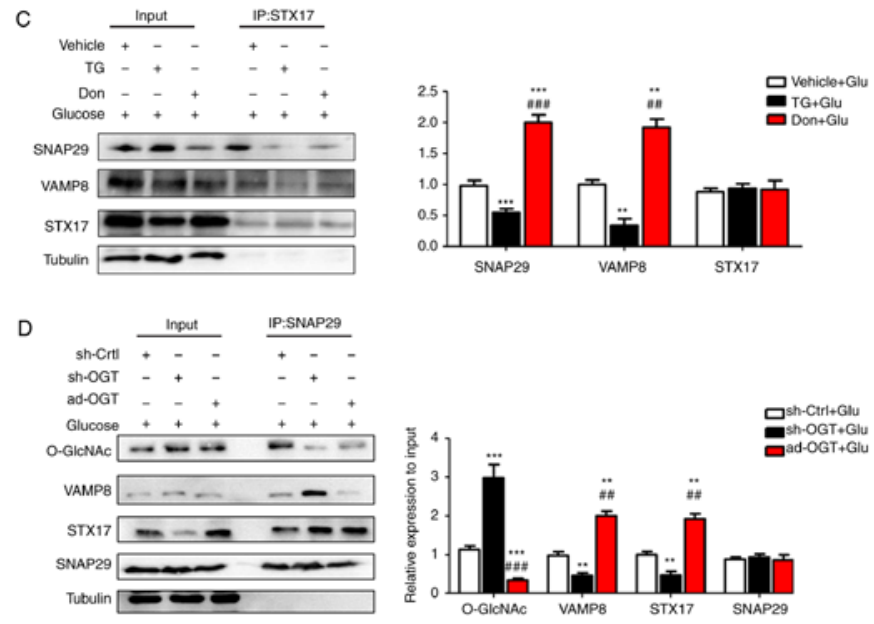
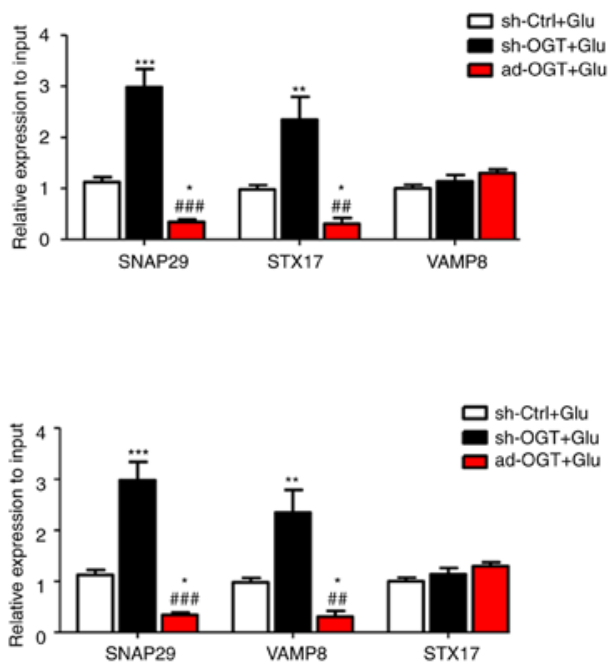

Figure 6. O-GlcNAc modification of SNAP29 inhibits SNAP29-STX17-VAMP8 complex formation and inhibits autophagy-mediated degradation. NRCMs were exposed to high glucose $(25 \mathrm{mM})$ and were treated with TG $(5 \mu \mathrm{M})$ or Don $(40 \mu \mathrm{M})$ for $24 \mathrm{~h}$. The combination of O-GlcNAc-modified SNAP29 into the SNAP29-STX17-VAMP8 complex was observed by co-IP. (A) SNAP29 antibody was used as pull down the VAMP8 and STX17, SNAP29 and Tubulin were used as the loading control; (B) VAMP8 antibody was applied to pull down the complex SNAP29 and STX17, VAMP8 and Tubulin were used as the loading control. (C) STX17 antibody was used to pull down the complex SANP29 and VAMP8, STX17 and Tubulin acted as the loading control. $\left({ }^{* *} \mathrm{P}<0.01,{ }^{* * * *} \mathrm{P}<0.001\right.$ vs. Vehicle+Glu group; ${ }^{\# \#} \mathrm{P}<0.01,{ }^{\# \# \#} \mathrm{P}<0.001$ vs. TG+Glu group); NRCMs were transfected with sh-OGT or ad-OGA for $48 \mathrm{~h}$ and to high glucose for another $24 \mathrm{~h}$, following which the formation of the SNAP29-STX17-VAMP8 complex was observed by co-IP. (D) SNAP29 antibody was used as pull down the VAMP8 and STX17, SNAP29 and Tubulin were used as the loading control; (E) VAMP8 antibody was applied to pull down the complex SNAP29 and STX17, VAMP8 and Tubulin were used as the loading control. (F) STX17 antibody was used to pull down the complex SANP29 and VAMP8, STX17 and Tubulin acted as the loading control. ( $\left(\mathrm{P}<0.05,{ }^{* *} \mathrm{P}<0.01,{ }^{* * *} \mathrm{P}<0.001\right.$ vs. sh-Ctrl+Glu group; ${ }^{\# / P} \mathrm{P}<0.01$, ${ }^{\# \# \#} \mathrm{P}<0.001$ vs. sh-OGT+Glu group); NRCMs, neonatal rat cardiomyocytes; O-GlcNAc, O-linked $\beta$-N-acetylglucosamine; SNAP29, synaptosomal-associated protein 29; vesicle-associated membrane protein 8; STX17, syntaxin-17; Don, 6-diazo-5-oxo-L-norleucine; TG, thiamet G; OGT, O-GlcNAc transferase; sh-OGT, OGT-knockdown adenovirus; ad-OGA, OGA-overexpression adenovirus; Ctrl, control; co-IP, coimmunoprecipitation.

cardiomyocytes exhibit different autophagic statuses due to inconsistent detection methods. Studies have suggested that the oxidation rates of fatty acids, glucose, and lactate are decreased in type I DM. Furthermore, insufficient energy due to low levels of ATP causes a cellular starvation state, and accumulated adenosine monophosphate (AMP) induces an increase in autophagy and myocardial injury by activating the AMP-activated protein kinase pathway (37). However, in a study by Kanamori et al (25), reduced autophagy was observed in the heart tissues of type I DM mice, and the administration of metformin prevented high glucose-induced myocardial injury by activating myocardial autophagy. Studies $(38,39)$ have demonstrated that STZ-induced type I DM mice exhibit autophagosome accumulation in the heart. The overactivation or inhibition of any step of autophagy can cause myocardial injury. The results of the present study also showed autophagosome accumulation in the STZ-induced type I DM rats and the significant inhibition of autophagic flow.

The O-GlcNAc modification and autophagy are regulated by high glucose, and the O-GlcNAc modification can regulate a variety of pathophysiological processes, including 


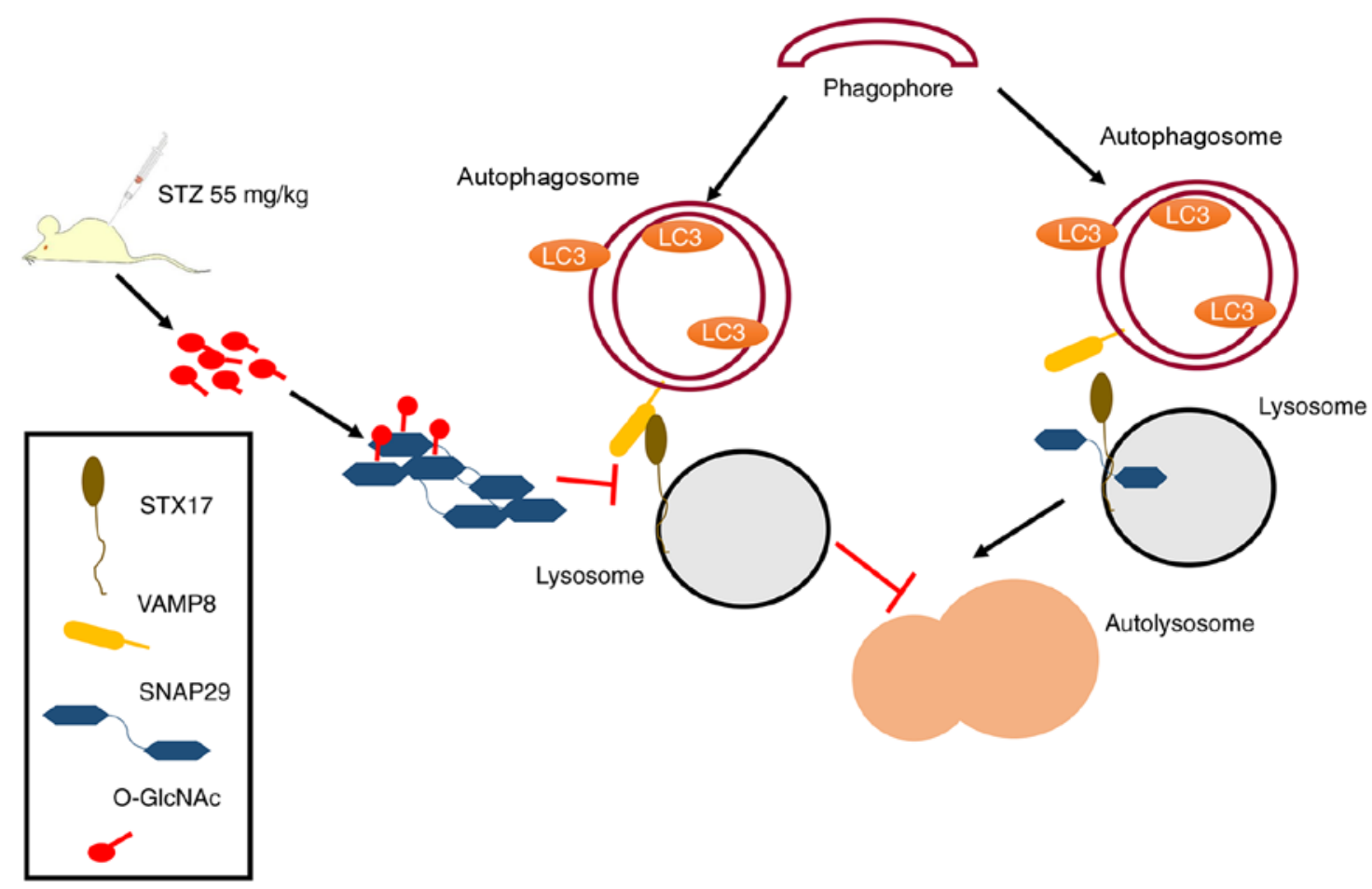

Figure 7. Schematic of the present study, including a description of the mechanism. Under normal circumstances, SNAP29 combines with SXT17 and VAMP8, forming a complex that is involved in the process of autophagosome and lysosome formation. In STZ-induced type I DM, the increase in O-GlcNAc-modified SNAP29 inhibits the formation of the SNAP29-STX17-VAMP8 complex, subsequently affecting autophagosome and lysosomal membrane fusion, which triggers myocardial injury in type I DM. O-GlcNAc, O-linked $\beta$-N-acetylglucosamine; SNAP29, synaptosomal-associated protein 29; vesicle-associated

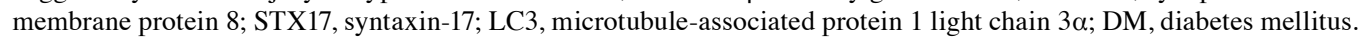

autophagy. The results of the present study showed that the autophagic flux of cardiomyocytes was significantly inhibited under high-glucose conditions, with enhancement of the $\mathrm{O}-\mathrm{GlcNAc}$ modification in vivo and in vitro. The present study also verified that the process of autophagic flux was negatively regulated by the O-GlcNAc modification in type I DM. The results of the present study demonstrated for the first time, to the best of our knowledge, that the formation of autophagosomes in cardiomyocytes was not affected by changes in the O-GlcNAc modification; however, the degradation stage of autophagy was affected by enhancement of the O-GlcNAc modification.

The regulation of autophagy by the O-GlcNAc modification is achieved by activating a substrate protein. As shown in previous studies $(22,40,41)$, membrane anchoring and fusion are central to the release of neurotransmitters by vesicles, but also in the process of membrane fusion between autophagosomes and lysosomes and the degradation in autophagic flux. The release of neurotransmitters is regulated mainly by SNARE protein complexes to ensure the accurate localization and fusion of the membrane. Therefore, SNARE complexes may be simultaneously involved in the regulation of the fusion between autophagosomes and lysosomes. The newly identified SNAP29 can bind to multiple syntaxin family members (STX7, STX8, and STX17) and is subsequently distributed on the plasma membrane and in the cytoplasm $(19,20)$. The SNAP29-STX17-VAMP8 complex mediates the membrane fusion process between autophagosomes and lysosomes. Studies (40-43) on SNAP29-mediated autophagy have mainly focused on the fruit fly, zebrafish, nematode and other biological models. In another previous study (44), siRNA-mediated silencing of STX17 in cultured human cells was found to result in a disrupted SNAP29-STX17-VAMP8 complex and inhibited autophagic flux.

However, no single study on SNAP29-mediated autophagy has been reported in mammalian cardiomyocytes or in disease models. The present study is the first, to the best of our knowledge, to demonstrate that SNAP29 is modified by $\mathrm{O}-\mathrm{GlcNAc}$ in rat cardiomyocytes. Under high-glucose conditions, O-GlcNAc-modified SNAP29 was increased in NRCMs, impeding the formation of the SNAP29-STX17-VAMP8 complex and consequently inhibiting autophagic flux. By contrast, a reduction in O-GlcNAc-modified SNAP29 promoted the formation of the SNAP29-STX17-VAMP8 complex and mediated degradation via autophagic flux. Therefore, the results of the present study revealed that O-GlcNAc-modified SNAP29 was increased and that the formation of the SNAP29-STX17-VAMP8 complex, which is involved in autophagy, was impaired in type I DM. The findings also demonstrated the inhibition of autophagic degradation as the underlying mechanism of myocardial injury in type I DM rats.

Although the present study demonstrated that O-GlcNAc-modified SNAP29 inhibited autophagy-mediated degradation in DM-related myocardial injury, the specific site of O-GlcNAc modification in SNAP29 requires further investigation. Furthermore, the incidence of type II DM is higher than that of type I DM, and the pathogenesis and autophagic state are not the same between type I and type II DM. The present study focused on the heart of type I DM 
rats; therefore, whether the results are generalizable to type II DM requires further investigation. The observed effect on the SNAP29-STX17-VAMP8 complex requires validation via protein knockdown experiments, to provide more evidence to support the findings of the present study.

In conclusion, O-GlcNAc-modified SNAP29 inhibited autophagic flux by inhibiting the formation of the SNAP29-STX17-VAMP8 complex, which is involved in the process of myocardial injury in type I DM. Elucidation of this mechanism clarifies the regulatory mechanism between the O-GlcNAc modification and autophagy in myocardial injury in type I DM rats. The inhibition of O-GlcNAc-modified SNAP29 can improve autophagic flux; therefore, O-GlcNAc-modified SNAP29 may be a potential therapeutic target for DM-related myocardial injury.

\section{Acknowledgements}

Not applicable.

\section{Funding}

The present study was supported by the National Natural Science Foundation of China (grant nos. 81400188 to JZH, 81360031 to YHT, 81500257 to RW and 81860152 to LH), the Natural Science Foundation of Jiangxi (grant nos. 20151BBB70266 and 20161BAB215242 to KH and LH, respectively) and the Innovation Fund Project in Jiangxi Province (grant no. YC2016-B020 to PiY).

\section{Availability of data and materials}

All data generated or analyzed during this study are included in this published article.

\section{Authors' contributions}

LH and PiY analyzed the data and wrote the manuscript; PeY, QK and ZX looked after the animals and performed western blot and co-IP analyses. YS performed the echocardiography and XY performed the histological examination of the hearts. JY and RW were primarily responsible for primary neonatal rat cardiomyocyte isolation and cell culture, and managed the drug treatments. KH performed data analysis and contributed to image editing. YT and JH conceived and designed the study, and gave the final approval of the version to be published. All authors have read and approved the final manuscript.

\section{Ethics approval and consent to participate}

All animal experiments were approved by the Animal Ethics and Experimentation Committee of Nanchang University and were performed in accordance with the 'Guide for the Care and Use of Laboratory Animals' (revised 1996). The experimental protocol was approved by the Second Affiliated Hospital of Nanchang University.

\section{Patient consent for publication}

Not applicable.

\section{Competing interests}

The authors declare that they have no competing interests.

\section{References}

1. Huynh K, Bernardo BC, McMullen JR and Ritchie RH: Diabetic cardiomyopathy: Mechanisms and new treatment strategies targeting antioxidant signaling pathways. Pharmacol Ther 142: 375-415, 2014.

2. Chavali V, Tyagi SC and Mishra PK: Predictors and prevention of diabetic cardiomyopathy. Diabetes Metab Syndr Obes 6: 151-160, 2013.

3. Dei CA, Khan SS, Butler J, Mentz RJ, Bonow RO, Avogaro A, Tschoepe D, Doehner W, Greene SJ, Senni M, et al: Impact of diabetes on epidemiology, treatment, and outcomes of patients with heart failure. JACC Heart Fail 3: 136-145, 2015.

4. Yi W, Clark PM, Mason DE, Keenan MC, Hill C, Goddard WA III, Peters EC, Driggers EM and Hsieh-Wilson LC: Phosphofructokinase 1 glycosylation regulates cell growth and metabolism. Science 337: 975-980, 2012.

5. Wang P and Hanover JA: Nutrient-driven O-GlcNAc cycling influences autophagic flux and neurodegenerative proteotoxicity. Autophagy 9: 604-606, 2013.

6. Pekkurnaz G, Trinidad JC, Wang X, Kong D and Schwarz TL: Glucose regulates mitochondrial motility via Milton modification by O-GlcNAc transferase. Cell 158: 54-68, 2014.

7. Ruan HB, Dietrich MO, Liu ZW, Zimmer MR, Li MD, Singh JP, Zhang K, Yin R, Wu J, Horvath TL and Yang X: O-GlcNAc transferase enables AgRP neurons to suppress browning of white fat. Cell 159: 306-317, 2014.

8. Peng C, Zhu Y, Zhang W, Liao Q, Chen Y, Zhao X, Guo Q, Shen P, Zhen B, Qian X, et al: Regulation of the Hippo-YAP pathway by glucose sensor O-GlcNAcylation. Mol Cell 68: 591-604, 2017.

9. Ngoh GA, Facundo HT, Zafir A and Jones SP: O-GlcNAc signaling in the cardiovascular system. Circ Res 107: 171-185, 2010.

10. Zhang Z, Costa FC, Tan EP, Bushue N, DiTacchio L, Costello CE, McComb ME, Whelan SA, Peterson KR and Slawson C: O-Linked N-Acetylglucosamine (O-GlcNAc) Transferase and $\mathrm{O}-$ GlcNAcase Interact with Mi2 $\beta$ Protein at the A $\gamma$-Globin Promoter. J Biol Chem 291: 15628-15640, 2016.

11. Erickson JR, Pereira L, Wang L, Han G, Ferguson A, Dao K, Copeland RJ, Despa F, Hart GW, Ripplinger CM and Bers DM: Diabetic hyperglycaemia activates CaMKII and arrhythmias by O-linked glycosylation. Nature 502: 372-376, 2013.

12. Park MJ, Kim DI, Lim SK, Choi JH, Han HJ, Yoon KC and Park SH: High glucose-induced O-GlcNAcylated carbohydrate response element-binding protein (ChREBP) mediates mesangial cell lipogenesis and fibrosis: The possible role in the development of diabetic nephropathy. J Biol Chem 289: 13519-13530, 2014.

13. Xie S, Jin N, Gu J, Shi J, Sun J, Chu D, Zhang L, Dai CL, Gu JH, Gong CX, et al: O-GlcNAcylation of protein kinase A catalytic subunits enhances its activity: A mechanism linked to learning and memory deficits in Alzheimer's disease. Aging Cell 15: 455-464, 2016.

14. Ma J and Hart GW: Protein O-GlcNAcylation in diabetes and diabetic complications. Expert Rev Proteomics 10: 365-380, 2013.

15. Banerjee PS, Ma J and Hart GW: Diabetes-associated dysregulation of O-GlcNAcylation in rat cardiac mitochondria. Proc Natl Acad Sci USA 112: 6050-6055, 2015.

16. Hu Y, Belke D, Suarez J, Swanson E, Clark R, Hoshijima M and Dillmann WH: Adenovirus-mediated overexpression of $\mathrm{O}-$ GlcNAcase improves contractile function in the diabetic heart. Circ Res 96: 1006-1013, 2005.

17. Gustafsson AB and Gottlieb RA: Recycle or die: The role of autophagy in cardioprotection. J Mol Cell Cardiol 44: 654-661, 2008.

18. Yorimitsu T and Klionsky DJ: Autophagy: Molecular machinery for self-eating. Cell Death Differ 12 (Suppl 2): S1542-S1552, 2005.

19. Hohenstein AC and Roche PA: SNAP-29 is a promiscuous syntaxin-binding SNARE. Biochem Biophys Res Commun 285: 167-171, 2001.

20. Diao J, Liu R, Rong Y,Zhao M, Zhang J,Lai Y, Zhou Q, Wilz LM, Li J, Vivona S, et al: ATG14 promotes membrane tethering and fusion of autophagosomes to endolysosomes. Nature 520: 563-566, 2015 . 
21. Bernard A and Klionsky DJ: Toward an understanding of autophagosome-lysosome fusion: The unsuspected role of ATG14. Autophagy 11: 583-584, 2015.

22. Liu R, Zhi X and Zhong Q: ATG14 controls SNARE-mediated autophagosome fusion with a lysosome. Autophagy 11: 847-849, 2015.

23. Guo B, Liang Q, Li L, Hu Z, Wu F, Zhang P, Ma Y, Zhao B, Kovács AL, Zhang Z, et al: O-GlcNAc-modification of SNAP-29 regulates autophagosome maturation. Nat Cell Biol 16: 1215-1226, 2014.

24. Bell RC, Carlson JC, Storr KC, Herbert K and Sivak J: High-fructose feeding of streptozotocin-diabetic rats is associated with increased cataract formation and increased oxidative stress in the kidney. Br J Nutr 84: 575-582, 2000.

25. Kanamori H, Takemura G, Goto K, Tsujimoto A, Mikami A Ogino A, Watanabe T, Morishita K, Okada H, Kawasaki M, et al: Autophagic adaptations in diabetic cardiomyopathy differ between type 1 and type 2 diabetes. Autophagy 11: 1146-1160, 2015.

26. Barefield DY, Puckelwartz MJ, Kim EY, Wilsbacher LD, Vo AH, Waters EA, Earley JU, Hadhazy M, Dellefave-Castillo L, Pesce LL and McNally EM: Experimental modeling supports a role for MyBP-HL as a Novel myofilament component in arrhythmia and dilated cardiomyopathy. Circulation 136: 1477-1491, 2017.

27. Riha H, Papoušek F, Neckář J, Pirk J and Oštádal B: Effects of isoflurane concentration on basic echocardiographic parameters of the left ventricle in rats. Physiol Res 61: 419-423, 2012.

28. Reinecke H, Zhang M, Bartosek T and Murry CE: Survival, integration, and differentiation of cardiomyocyte grafts: A study in normal and injured rat hearts. Circulation 100: 193-202, 1999.

29. Peng X, Shao J, Shen Y, Zhou Y, Cao Q, Hu J, He W, Yu X, Liu X, Marian AJ and Hong K: FAT10 protects cardiac myocytes against apoptosis. J Mol Cell Cardiol 59: 1-10, 2013.

30. Mellor KM, Bell JR, Young MJ, Ritchie RH and Delbridge LM: Myocardial autophagy activation and suppressed survival signaling is associated with insulin resistance in fructose-fed mice. J Mol Cell Cardiol 50: 1035-1043, 2011.

31. Tanida I, Wakabayashi M, Kanematsu T, Minematsu-Ikeguchi N, Sou YS, Hirata M, Ueno T and Kominami E: Lysosomal turnover of GABARAP-phospholipid conjugate is activated during differentiation of $\mathrm{C} 2 \mathrm{C} 12$ cells to myotubes without inactivation of the mTor kinase-signaling pathway. Autophagy 2: 264-271, 2006.

32. Darley-Usmar VM, Ball LE and Chatham JC: Protein O-linked $\beta$-N-acetylglucosamine: A novel effector of cardiomyocyte metabolism and function. J Mol Cell Cardiol 52: 538-549, 2012.

33. Jesmin S, Zaedi S, Shimojo N, Iemitsu M, Masuzawa K, Yamaguchi N, Mowa CN, Maeda S, Hattori Y and Miyauchi T: Endothelin antagonism normalizes VEGF signaling and cardiac function in STZ-induced diabetic rat hearts. Am J Physiol Endocrinol Metab 292: E1030-E1040, 2007.

34. Chen ZC, Cheng YZ, Chen LJ, Cheng KC, Li Y and Cheng J: Increase of ATP-sensitive potassium (K(ATP)) channels in the heart of type-1 diabetic rats. Cardiovasc DiabetoL 11: 8, 2012.
35. Li HT, Wu XD, Davey AK and Wang J: Antihyperglycemic effects of baicalin on streptozotocin-nicotinamide induced diabetic rats. Phytother Res 25: 189-194, 2011.

36. Sun D, Shen M, Li J, Li W, Zhang Y, Zhao L, Zhang Z, Yuan Y, Wang $\mathrm{H}$ and Cao F: Cardioprotective effects of tanshinone IIA pretreatment via kinin B2 receptor-Akt-GSK-3 $\beta$ dependent pathway in experimental diabetic cardiomyopathy. Cardiovase Diabetol 10: 4,2011.

37. Qiao L, Guo B, Zhang H, Yang R, Chang L, Wang Y, Jin X, Liu S and Li Y: The clock gene, brain and muscle Arnt-like 1, regulates autophagy in high glucose-induced cardiomyocyte injury. Oncotarget 8: 80612-80624, 2017.

38. Xie Z, Lau K, Eby B, Lozano P, He C, Pennington B, Li H, Rathi S, Dong Y, Tian R, et al: Improvement of cardiac functions by chronic metformin treatment is associated with enhanced cardiac autophagy in diabetic OVE26 mice. Diabetes 60: 1770-1778, 2011.

39. Wang B, Yang Q, Sun YY, Xing YF, Wang YB, Lu XT, Bai WW, Liu XQ and Zhao YX: Resveratrol-enhanced autophagic flux ameliorates myocardial oxidative stress injury in diabetic mice. J Cell Mol Med 18: 1599-1611, 2014.

40. Li Q, Frank M, Akiyama M, Shimizu H, Ho SY, Thisse C, Thisse B, Sprecher E and Uitto J: Abca12-mediated lipid transport and Snap29-dependent trafficking of lamellar granules are crucial for epidermal morphogenesis in a zebrafish model of ichthyosis. Dis Model Mech 4: 777-785, 2011.

41. Morelli E, Ginefra P, Mastrodonato V, Beznoussenko GV, Rusten TE, Bilder D, Stenmark H, Mironov AA and Vaccari T: Multiple functions of the SNARE protein Snap29 in autophagy, endocytic, and exocytic trafficking during epithelial formation in Drosophila. Autophagy 10: 2251-2268, 2014.

42. Jiu Y, Hasygar K, Tang L, Liu Y, Holmberg CI, Bürglin TR, Hietakangas V and Jäntti J: par-1, atypical pkc, and PP2A/B55 sur-6areimplicated in the regulation of exocyst-mediated membrane trafficking in Caenorhabditis elegans. G3 (Bethesda) 4: 173-183, 2014.

43. Sato M, Saegusa K, Sato K, Hara T, Harada A and Sato K: Caenorhabditis elegans SNAP-29 is required for organellar integrity of the endomembrane system and general exocytosis in intestinal epithelial cells. Mol Biol Cell 22: 2579-2587, 2011.

44. Itakura E, Kishi-Itakura C and Mizushima N: The hairpin-type tail-anchored SNARE syntaxin 17 targets to autophagosomes for fusion with endosomes/lysosomes. Cell 151: 1256-1269, 2012.

This work is licensed under a Creative Commons Attribution-NonCommercial-NoDerivatives 4.0 International (CC BY-NC-ND 4.0) License. 